\title{
On the Viscous Cahn-Hilliard-Oono System with Chemotaxis and Singular Potential
}

\author{
Jingning $\mathrm{He}^{1}$ \\ ${ }^{1}$ Fudan University - Handan Campus
}

May 29, 2021

\begin{abstract}
We analyze a diffuse interface model that couples a viscous Cahn-Hilliard equation for the phase variable with a diffusion-reaction equation for the nutrient concentration. The system under consideration also takes into account some important mechanisms like chemotaxis, active transport as well as nonlocal interaction of Oono's type. When the spatial dimension is three, we prove the existence and uniqueness of global weak solutions to the model with singular potentials including the physically relevant logarithmic potential. Then we obtain some regularity properties of the weak solutions when $t>0$. In particular, with the aid of the viscous term, we prove the so-called instantaneous separation property of the phase variable such that it stays away from the pure states \pm 1 as long as $\mathrm{t}>0$. Furthermore, we study long-time behavior of the system, by proving the existence of a global attractor and characterizing its $\omega$-limit set.
\end{abstract}

\section{Hosted file}

Viscous-CHO-20210526.pdf available at https://authorea.com/users/416559/articles/524058-onthe-viscous-cahn-hilliard-oono-system-with-chemotaxis-and-singular-potential 